\title{
PROPUESTA DIDÁCTICA PARA ESTIMULAR EL EQUILIBRIO EN PATINADORES DE CARRERAS MEDIANTE LA PROPIOCEPCIÓN
}

Didactic proposal to stimulate the balance in race skaters through proprioception

DIEGO ARMANDO DÍAZ RODRÍGUEZ dte_dadiazr924@pedagogica.edu.co

JUAN DAVID GASCA RODRÍGUEZ fef_jdgascar409@pedagogica.edu.co

DAVID ESTABAN ROJAS LEGUIZAMÓN fef_derojasl462@pedagogica.edu.co

BORYI ALEXANDER BECERRA PATIÑO (Docente investigador) babecerrap@pedagogica.edu.co

Universidad Pedagógica Nacional (Colombia)

Artículo de investigación formativa

Recepción: 21 de noviembre de 2020

Aceptación: 21 de abril de 2021 


\section{Resumen:}

La situación a causa del Covid-19 obligó a los entes estatales a generar estrategias de contención del virus, trayendo afectaciones en el entrenamiento deportivo presencial. Por esta razón, el presente trabajo tiene como propósito desarrollar una propuesta didáctica para el entrenamiento del equilibrio en patinadores de la categoría infantil (8 y 9 años) en la ciudad de Bogotá. Toma la propiocepción como eje dinamizador que apoye al trabajo en casa, a partir de la utilización de material reciclable o de fácil acceso. Esta investigación ha sido planteada a partir de una metodología de investigación proyectiva, direccionada por los diferentes estadios de los que se constituye la propuesta. Se realizó una consulta de los antecedentes en bases de datos, repositorios y el buscador Google académico, mediante la combinación de palabras clave: propiocepción, patinaje y equilibrio. La propuesta metodológica se divide en tres (3) fases (adaptación, refinamiento y dominio), con 12 unidades. Finalmente, se ha encontrado que el entrenamiento propioceptivo puede producir mejoras en el equilibrio.

Palabras clave: deporte; pedagogía; patinaje.

\section{Abstract:}

The current situation because the Covid-19 forced estatal entities to generate strategies to control the virus, generating affectations in face-to-face sport training. For this reason, this study has the purpose to develop a didactical proposal to balance training in the infant category ( 8 and 9 years old) race skaters in Bogotá. Takes the proprioception as the dynamic axis that supports the training at home, from the use of recyclable material or easy access. This research has been raised using projective research, directed by the different stadiums which the proposal is constituted. A query was made of the background in databases, repositories and academic Google, through the combination of keywords: proprioception, roller skate and equilibrium. This methodological proposal is divided in three (3) phases (adaptation, refinement and domain), with 12 units. Finally, it has found that the proprioceptive training can produce improvements in balance.

Keywords: sport; pedagogy; skating. 


\section{Introducción}

La emergencia sanitaria ocasionada por la pandemia de la Covid-19 ha cambiado los estilos de vida. Desde el deporte y la actividad física se ha generado diferentes estrategias para mitigar el impacto frente a la necesidad de proteger la salud, continuar con los entrenamientos y disminuir el impacto nocivo de esta enfermedad (Villaquiran, Ramos, Jácome y Mesa, 2020). Esta investigación proyectiva consiste en la elaboración de una propuesta didáctica, mediante una guía de entrenamientos para el patinaje de carreras en ciudad de Bogotá. Allí, a través del entrenamiento en casa y apoyados de material reciclable, los deportistas podrán entrenar el equilibrio por medio de la propiocepción, siendo este un aspecto fundamental en el patinaje (Pinzón, 2017).

La técnica en el patinaje de carreras es fundamental; como plantea Lugea (2009), es una disciplina muy táctica, donde juega un papel importante las leyes de la mecánica y las características individuales del deportista (Martínez, 1991). Entonces, la técnica puede entrenarse a través de diferentes medios con el fin de obtener mejoras y resultados deportivos positivos (Narváez, 2005; Weineck, 2005).

Por otro lado, cabe recalcar que el entrenamiento propioceptivo realizado de una forma adecuada genera mejoras en el equilibrio en patinadores de carreras (García y Rodríguez, 2015; Egocheaga et al., 2004). Además, proporciona una mejor técnica y capacidad de reacción en los deportistas (Bolaños, 2010). El entrenamiento del equilibrio y la propiocepción, principalmente ha sido en rehabilitación de lesiones (Pinzón, Vidarte y Sánchez, 2019) y no tanto hacia la mejora físico-táctica, por el contrario, hacia la coordinación y el equilibrio (Noreña, 2015; García y Rodríguez, 2015).

\section{Metodología}

La propuesta metodológica está compuesta por doce (12) unidades didácticas, respetando los estadios de la investigación proyectiva (Hurtado, 2015). Estas fases son las que guían el proceso de construcción del proyecto investigativo, dando una coherencia y consecución idónea para permitir el desarrollo correspondiente del diseño de la propuesta y sus características (Hurtado, 2000). 


\begin{tabular}{|l|l|}
\hline Descriptivo & $\begin{array}{l}\text { Mejoramiento del equilibrio en patinadores por me- } \\
\text { dio de ejercicios propioceptivos. }\end{array}$ \\
\hline Analítico & $\begin{array}{l}\text { Mejora de la capacidad del equilibrio por medio de } \\
\text { una propuesta metodológica que se adapte a la prác- } \\
\text { tica en casa, articulando con la sustentación teórica } \\
\text { pertinente. }\end{array}$ \\
\hline Comparativo & $\begin{array}{l}\text { Por medio de un rastreo bibliográfico se puede de- } \\
\text { terminar que es importante el desarrollo del equili- } \\
\text { brio en patinadores por medio de la propiocepción. }\end{array}$ \\
\hline Explicativo & $\begin{array}{l}\text { Desarrollar una propuesta didáctica para el desarro- } \\
\text { llo y comprensión de ejercicios propioceptivos para } \\
\text { aplicarlo en el contexto de patinaje de carreras. }\end{array}$ \\
\hline Predictivo & $\begin{array}{l}\text { Teniendo en cuenta la etapa de desarrollo de los de- } \\
\text { portistas infantiles de 8 - 9 años se espera mejorar el } \\
\text { nivel del equilibrio mediante la técnica de patinaje } \\
\text { de carreras. }\end{array}$ \\
\hline Proyectivo & $\begin{array}{l}\text { Diseñar una propuesta didáctica para estimular el } \\
\text { equilibrio en deportistas de patinaje por medio de } \\
\text { la formación de la habilidad motora, que permita la } \\
\text { ejecución técnica correcta del patinaje de carreras. }\end{array}$ \\
\hline
\end{tabular}

Tabla 1. Cuadro de estadios de la propuesta metodológica

En la formación de la habilidad motora, según Lugea (2009), es necesario distinguir tres (3) fases de formación:

1.Desarrollo de la coordinación general, que hace referencia a la adaptación del gesto técnico que pretende aprender.

2.Desarrollo de la coordinación fina, que exalta el movimiento.

3.Desarrollo de la estabilización de la coordinación fina, la consolidación y perfeccionamiento de la destreza del movimiento para el rendimiento deportivo.

Los ejercicios planteados posteriormente se presentan como una opción para que se puedan realizar en casa debido a la situación actual de la pandemia del Covid-19. Así, favorece la continuidad del entrenamiento, que por medio de la propiocepción permita la estimulación del equilibrio, por ende, también la reincorporación a competencia y ritmo de trabajo habituales en los deportistas luego de la contingencia, disminuyendo también los riesgos de lesión. 
Por otro lado, es importante recalcar la importancia que tiene el equilibrio en el deporte del patinaje de carreras, razón por la que es fundamental hacer ejercicios que conlleven a una mejora de esta capacidad. De esta manera, promover respuestas más eficaces y rápidas frente a las acciones que se presentan durante la competencia.

Un aspecto muy importante en los ejercicios propuestos en este trabajo es que se busca que los materiales requeridos para su realización puedan ser reciclables, con el fin de evitar un impacto negativo sobre el medio ambiente, o de fácil acceso para los deportistas. Así, favorece que la cantidad de personas que puedan llevarlos a cabo sea mayor, fomentando así la práctica y masificación del patinaje de carreras en nuestro país.

Teniendo en cuenta que actualmente la virtualidad se ha convertido en una parte fundamental para la sociedad; además, entendiendo que el deporte no es ajeno a esta transformación; a su vez, se busca adaptar esta temática a un nuevo paradigma, tomando la tecnología actual como los códigos QR, los medios audiovisuales, plataformas virtuales y portales de internet como Youtube. El objetivo es facilitar la difusión, garantizar la comunicación y el entendimiento de una buena técnica de ejecución, de cada uno de los ejercicios, ya que estas herramientas son de fácil acceso permitiendo así hacerse con dicha información.

\section{Entrenamiento propioceptivo}

El entrenamiento propioceptivo planteado adecuadamente puede generar mejoras en la coordinación, ritmo, mecanismos reflejos y sobre el equilibrio tanto estático como dinámico (Noreña, 2015). Esta última capacidad en especial puede verse bastante beneficiada; este entrenamiento busca proporcionar fuerzas o perturbaciones que afecten el balance de la persona, con el fin de producir respuestas neuromusculares que conlleven a compensar dichos factores externos y retomar un estado de equilibrio (Pinzón, 2017).

Por otro lado, Pinzón, Vidarte y Sánchez (2019) plantean que según estudios realizados por Brachman et al. (2017), en entrenamiento propioceptivo en atletas de siete (7) a 30 años debe realizarse de entre ocho (8) a 12 semanas con una frecuencia mínima de dos (2) veces por semana para que su efecto sea positivo.

En este tipo de entrenamiento es fundamental tener una progresión desde ejercicios sencillos (Noreña, 2015) que inicien realizándose con los ojos abiertos, apoyo bipodal sobre una base estable planteando posturas adecuadas. Permiten un equilibrio con una buena base de sustentación y centro de gravedad bajo, para luego ir aumentando de dificultad poco a poco, con variables como cerrar los ojos, apoyo unipodal, bases inestables o realización de movimientos, entre otros (Pinzón, 2017).

A continuación, se presenta un ejemplo de una de las unidades didácticas que desarrolló el equipo de trabajo. Responde a la propuesta que busca además de una fácil difusión, acceso e implementación, la estimulación del equilibrio a través de ejercicios propioceptivos. 
Tabla 2. Ejemplo de la propuesta didáctica

\begin{tabular}{|c|c|}
\hline \multicolumn{2}{|c|}{ Ejercicio 1} \\
\hline & \\
\hline \multicolumn{2}{|c|}{ Descripción } \\
\hline \multicolumn{2}{|c|}{$\begin{array}{l}\text {-Paso 1: postura inicial, postura de patinador (flexión de rodillas en un ángu- } \\
\text { lo de } 90^{\circ} \text { respecto al piso). } \\
\text {-Paso 2: realice una abducción distal de la zona del gastrocnemio posterior } \\
\text { (pierna derecha o izquierda). } \\
\text {-Paso 3: mantener la postura de manera estática. }\end{array}$} \\
\hline Objetivo del ejercicio & Características \\
\hline $\begin{array}{l}\text { Mejorar la estabilidad en la postura de } \\
\text { patinador por medio de la propiocep- } \\
\text { ción, permitiendo un ajuste corporal } \\
\text { desplegado por el sistema nervioso } \\
\text { central, desarrollo del equilibrio y la- } \\
\text { teralidad. }\end{array}$ & $\begin{array}{l}\text {-Factor condicional: ojos cerrados. } \\
\text {-Acción articular: flexión de rodilla y } \\
\text { flexión de cadera. } \\
\text {-Musculatura principal: cuadro lum- } \\
\text { bar, recto abdominal, glúteo mayor, } \\
\text { psoas iliaco y semitendinoso. } \\
\text {-Grado de complejidad: medio. } \\
\text { Cadena cinética: cerrado. } \\
\text {-Elementos: no. }\end{array}$ \\
\hline Aportes & Aspectos a tener en cuenta \\
\hline $\begin{array}{l}\text {-Motriz: lateralidad, esquema corporaly } \\
\text { estructura espaciotemporal. } \\
\text {-Técnica: postura de patinador y reco- } \\
\text { bro del empuje. } \\
\text {-Tiempo: Se propone que se pueda rea- } \\
\text { lizar dos veces a la semana mínimo } 30 \\
\text { minutos con sus diferentes progresiones } \\
\text { y devoluciones. }\end{array}$ & $\begin{array}{l}\text {-Promover: realizar movimientos arti- } \\
\text { culares antes de realizar el ejercicio. } \\
\text {-Evitar: el pie que está elevado no toca } \\
\text { el suelo. } \\
\text {-Variable 1: Realizar el ejercicio con pie } \\
\text { derecho e izquierdo. }\end{array}$ \\
\hline
\end{tabular}




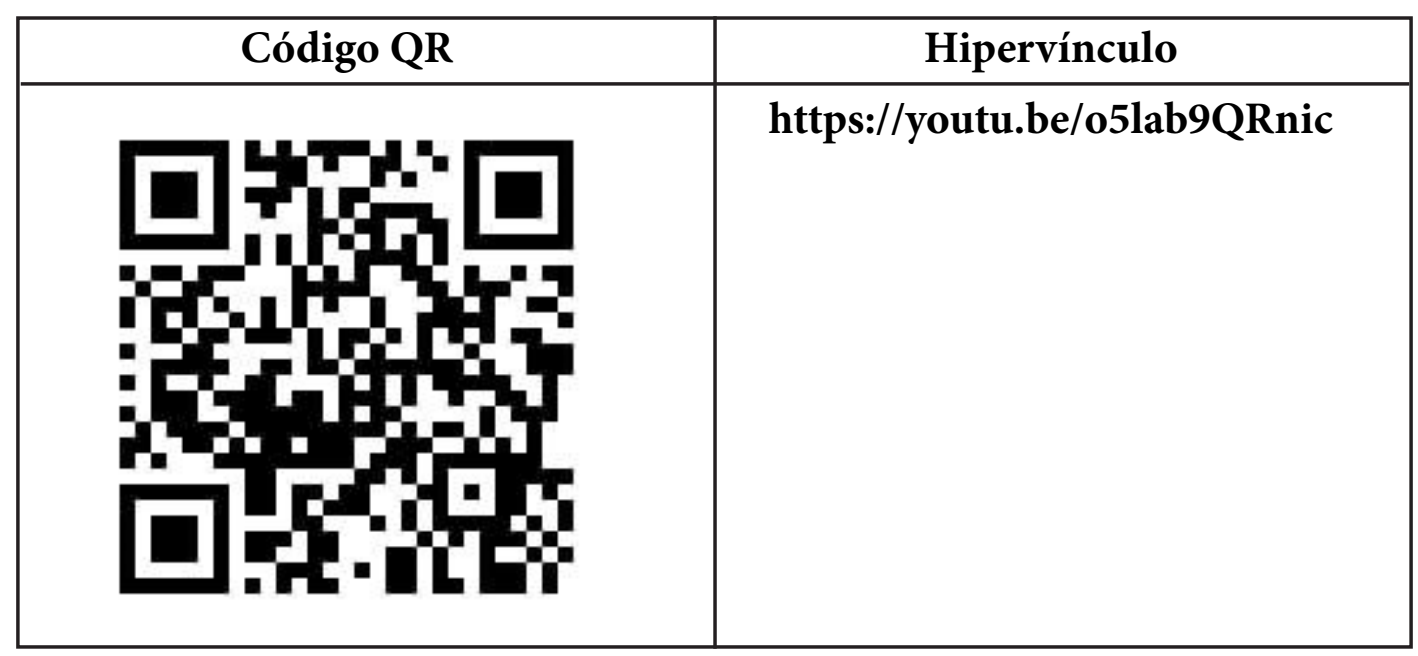

Fuente. Elaboración propia

\section{Resultados y discusión}

El equilibrio en el patinaje de carreras se puede estimular a través de ejercicios propioceptivos desarrollados en casa por medio de materiales didácticos, por el contexto actual de la pandemia del Covid-19. Sin embargo, no se encontraron a la fecha estudios evidencien el entrenamiento del patinaje en casa; hay evidencia de trabajos propioceptivos para la mejora del equilibrio como el de Pinzón (2017) y García y Rodríguez (2015).

Cabe destacar que los documentos hallados que hablan sobre el entrenamiento del equilibrio por medio de la propiocepción en el patinaje de carreras son pocos. No obstante, se encontraron también en referencia otros deportes, principalmente fútbol y balonmano. En los estudios de patinaje de carreras se evidenció una mejora frente al equilibrio de los deportistas (Pinzón, 2017; Pinzón et al., 2019).

Según Brachman et al. (2017), pese a que varios estudios demostraron que hubo una mejoría en el equilibrio gracias al trabajo propioceptivo, otros (en menor medida) obtuvieron resultados diferentes. Pudo haber pasado porque el tiempo de aplicación no fue homogéneo en todos los estudios Pese a esto, para Brachman et al. (2017) y Pinzón (2017) aquellos estudios que realizaron entrenamiento por 12 semanas, con una frecuencia de tres (3) veces por semana, arrojaron resultados positivos.

\section{Conclusiones}

Este trabajo es pionero en la elaboración de una propuesta didáctica de trabajo en casa en el entrenamiento del equilibrio, por medio de la propiocepción con herramientas tecnológicas de difusión, teniendo en cuenta su importancia en la situación actual. Por otro lado, los trabajos como estos ayudan al entrenamiento del patinaje desde casa y como plan de contingencia contra la emergencia sanitaria. 
Se ha encontrado que el entrenamiento propioceptivo puede producir mejoras en el equilibrio, favoreciendo la implementación de este tipo de trabajos, diseñados para favorecer la estimulación del equilibrio a través de la propiocepción. Además, respetando el principio pedagógico del entrenamiento de participación activa, consciente y especificidad, con ejercicios en casa para el patinaje de carreras. A su vez, responde a la necesidad del entrenamiento asincrónico, por la contingencia actual de la pandemia de la Covid-19.

Por tanto, dada la situación actual, la evidencia que se tiene de estudios anteriores con respecto a la mejora del equilibrio por medio de ejercicios propioceptivos y la facilidad de realizarlos con materiales asequibles para muchas personas, así como elaborarlos por medio de objetos reciclables; es una propuesta que puede llegar a tener un impacto positivo en este deporte. 


\section{Referencias bibliográficas}

Bolaños, D. (2010). Desarrollo motor, movimiento e interacción. Armenia: Kinesis.

Brachman, A., Kamieniarz, A., MichaIska, J., Powtowaki, M., Stomka, K. y Juras, G. (2017). Balance training program in athletes: a systematic review. Journal of human kinetics, 58, 45-64.

Egocheaga, J., Llavador, J., Díaz-Munío, J., Del Valle, M., Egia, O. y Díaz, I. (2004). Economía de carrera del patinador de velocidad en función de su posición dentro del grupo. Archivos de medicina del deporte. 21(101), 215-220.

García, J. y Rodríguez, J. (2015). Equilibrio y estabilidad del cuerpo humano. León, España: Universidad de León.

Hurtado, J. (2000). Metodología de la investigación holística. Caracas: Sypal.

Hurtado, J. (2015). Metodología de la investigación holística. Caracas: Sypal.

Lugea, C. (2009). Fundamentos de la técnica del patinaje de velocidad. spagatta.
Narváez, M. (2005). Características neurocognitivas y psicológicas de los patinadores de altos logros deportivos del departamento de Antioquia, en la modalidad de carreras (tesis). Medellín: Universidad San Buenaventura.

Noreña, M. (2015). Propiocepción con pelotas en niños en etapa escolar. Revista de Educación física de la Universidad de Antioquia, 4(4), 1-32.

Pinzón, S. (2017). Efectos de un programa de ejercicio físico propioceptivo sobre el equilibrio en jóvenes patinadores entre los 11 y 15 años pertenecientes a la Liga Santandereana de Patinaje de Carreras en la ciudad de Bucaramanga 2016 (tesis de maestría). Manizales: Universidad Autónoma de Manizales.

Pinzón, S., Vidarte, J. y Sánchez, J. (2019). Efectos de un programa de ejercicio físico propioceptivo sobre el equilibrio en jóvenes patinadores entre los 11 y 15 años. Archivos de Medicina de Deporte, 36(3), 166-171.

Villaquiran, A., Ramos, A., Jácome, J. y Mesa M. (2020). Actividad física y ejercicio en tiempos de covid-19. Ces Medicina, 34, 51-58. 